\title{
Identification of combinatorial host-specific signatures with a potential to affect host adaptation in influenza A H1N1 and H3N2 subtypes
}

Zeeshan Khaliq ${ }^{1}$, Mikael Leijon ${ }^{2,3}$, Sándor Belák ${ }^{3,4}$ and Jan Komorowski ${ }^{1,5^{*}}$

\begin{abstract}
Background: The underlying strategies used by influenza A viruses (IAVs) to adapt to new hosts while crossing the species barrier are complex and yet to be understood completely. Several studies have been published identifying singular genomic signatures that indicate such a host switch. The complexity of the problem suggested that in addition to the singular signatures, there might be a combinatorial use of such genomic features, in nature, defining adaptation to hosts.

Results: We used computational rule-based modeling to identify combinatorial sets of interacting amino acid (aa) residues in 12 proteins of IAVs of H1N1 and H3N2 subtypes. We built highly accurate rule-based models for each protein that could differentiate between viral aa sequences coming from avian and human hosts. We found 68 host-specific combinations of aa residues, potentially associated to host adaptation on HA, M1, M2, NP, NS1, NEP, PA, PA-X, PB1 and PB2 proteins of the H1N1 subtype and 24 on M1, M2, NEP, PB1 and PB2 proteins of the H3N2 subtypes. In addition to these combinations, we found 132 novel singular aa signatures distributed among all proteins, including the newly discovered PA-X protein, of both subtypes. We showed that HA, NA, NP, NS1, NEP, PA-X and PA proteins of the H1N1 subtype carry H1N1-specific and HA, NA, PA-X, PA, PB1-F2 and PB1 of the H3N2 subtype carry H3N2-specific signatures. M1, M2, PB1-F2, PB1 and PB2 of H1N1 subtype, in addition to H1N1 signatures, also carry H3N2 signatures. Similarly M1, M2, NP, NS1, NEP and PB2 of H3N2 subtype were shown to carry both H3N2 and H1N1 host-specific signatures (HSSs).
\end{abstract}

Conclusions: To sum it up, we computationally constructed simple IF-THEN rule-based models that could distinguish between aa sequences of avian and human IAVs. From the rules we identified HSSs having a potential to affect the adaptation to specific hosts. The identification of combinatorial HSSs suggests that the process of adaptation of IAVs to a new host is more complex than previously suggested. The present study provides a basis for further detailed studies with the aim to elucidate the molecular mechanisms providing the foundation for the adaptation process.

Keywords: Influenza A virus, Host adaptation, Combinatorial signatures, Host-specific signatures, MCFS, Rosetta, Rough sets

\footnotetext{
* Correspondence: jan.komorowski@icm.uu.se

'Department of Cell and Molecular Biology, Computational Biology and

Bioinformatics, Science for Life Laboratory, Uppsala University, SE-751 24 Uppsala, Sweden

${ }^{5}$ Institute of Computer Science, Polish Academy of Sciences, 01-248

Warszawa, Poland

Full list of author information is available at the end of the article
} 


\section{Background}

Influenza A viruses (IAVs) have been known for a long time to cause disease in a wide range of host species, including humans and various animals. The IAVs are zoonotic pathogens that can infect a broad range of animals from birds to pigs and humans. The interspecies transmission requires that IAVs adapt to the new host and the whole process is facilitated by their high mutation rates [1] and their ability to readily reassort [2]. This can result in epidemics and pandemics with severe consequences for both human and animal life. In addition to the yearly epidemics that proves fatal for at least 250,000 humans worldwide [3], in the 20th century alone, there has been at least five major pandemics; the Spanish flu of 1918, Asian influenza of 1957, Hong Kong influenza of 1968, the age restricted milder Russian flu of the 1977 [4, 5] and the Swine flu of 2009. Thus, new flu epidemics and pandemics are a constant threat. Given our poor understanding of the host adaptation process of the virus, which can be a major factor for such epidemics and pandemics, it is very hard to predict the type of the virus that will cause the coming outbreaks.

The IAVs are usually classified into subtypes based on the two surface glycol-proteins, hemagglutinin (HA) and neuraminidase (NA). To date, 18 types of HA (H1-H18) and 11 types of NA (N1-N11) are known [6-8]. Most of these subtypes have wild birds as their natural hosts. However, occasionally the virus can jump and adapt to a new host species. This cross of the species barrier is proved by the pandemic $\mathrm{H} 1 \mathrm{~N} 1, \mathrm{H} 3 \mathrm{~N} 2, \mathrm{H} 2 \mathrm{~N} 2$ and the most recent $\mathrm{H} 5 \mathrm{~N} 1$ and $\mathrm{H} 7 \mathrm{~N} 9$ subtype outbreaks, which are thought to have evolved from avian or porcine sources $[6,9,10]$.

The HA protein plays a crucial part in defining the adaptation of the virus to different hosts since it binds to the receptor providing the entry into host cells. The avian strains of the IAVs are known to attach to a receptor with $\alpha 2,3$-sialic acid linkages while the human strains to a receptor with $\alpha 2,6$-sialic acid linkages [11]. However, other proteins such as the polymerase subunits have also previously been shown to play a role in the adaptation of IAVs to different hosts $[12,13]$.

Computational methods, like artificial neural networks, support vector machines and random forests, have been used previously to predict hosts of IAVs [14-16]. Furthermore, several other studies have previously been carried out predicting genomic signatures specifying different hosts, both computationally and experimentally [17-23]. Amino acid changes taken one at a time, i.e. singular aa changes, in viral protein sequences between different hosts have been reported by these studies as host-specific signatures (HSSs), some of which likely facilitate the host adaptation process. Despite these findings, the process of adaptation of
IAVs in different hosts is still not completely understood. Given the complex nature of the problem we suspected that the adaptation process might not only be dependent on univariate signatures. Essentially, in addition to the proven effects of singular aa residues, there might be a combinatorial use of aa residues in nature that affect the adaptation of IAVs to new hosts.

To this end, for both H1N1 and H3N2 subtypes, we analyzed aa sequences of 12 proteins expressed by the viruses. We have restricted our analyses to these two subtypes because data for both human and avian hosts for all the proteins under-study was available. We built high quality rule-based models, based on rough sets [24], for each of the 12 proteins, predicting hosts from protein sequences. The models consisted of simple IFTHEN rules that lend themselves to easy interpretation. The combinations of aa residues used by the rules were identified as host-specific signatures having the potential to affect the host adaptation of IAVs. In additions to such combinatorial signatures, novel singular signatures were also identified from the rules. The singular and, especially, the combinatorial signatures provide novel insights into the complex host adaptation process of the IAVs.

\section{Results}

Feature selection reduces the number of features needed to discern between hosts

Monte Carlo Feature Selection (MCFS) [25] was used to obtain a ranked list of significant features, here significantly informative aa positions in all the proteins for both subtypes, that best discern between the hosts. This step helped us remove any kind of noise that could have been in the data. More importantly, the use of MCFS considerably reduced the number of aa positions to be analyzed further, as shown in Table 1 . The HA protein had 628 positions to start with and after running MCFS on the data, we were left with 115 and 88 positions for H1N1 and H3N2 subtypes, respectively (81.7 and $86 \%$ reduction in the number aa positions). On average there was a $79.8 \%$ reduction in the number of aa positions across all the proteins for H1N1 subtype and $82.8 \%$ for the H3N2 subtype (Table 1). Only the significant features were used for further analysis in this study. The ranked lists of the significant features are provided as a supplementary file (see Additional file 1).

\section{Rule-based models for each protein}

Since the number of sequences belonging to human and avian hosts were not balanced in the training data of either subtype (Table 1), we balanced the data sets by a method called under-sampling, as described in detail in Methods. For data sets of each protein and each subtype we created 100 under-sampled subsets. Each of these 
Table 1 The training data

\begin{tabular}{|c|c|c|c|c|c|c|c|}
\hline \multirow[b]{3}{*}{ Protein } & \multicolumn{4}{|c|}{ Nr. of sequences for each subtype } & \multirow[b]{3}{*}{ Total features } & \multirow{2}{*}{\multicolumn{2}{|c|}{ Features after MCFS }} \\
\hline & \multicolumn{2}{|l|}{$\mathrm{H} 1 \mathrm{~N} 1$} & \multicolumn{2}{|l|}{$\mathrm{H} 3 \mathrm{~N} 2$} & & & \\
\hline & Avian & Human & Avian & Human & & H1N1 & $\mathrm{H} 3 \mathrm{~N} 2$ \\
\hline $\mathrm{HA}$ & 214 & 5205 & 164 & 3715 & 628 & 115 & 88 \\
\hline NA & 205 & 3093 & 173 & 3412 & 517 & 93 & 79 \\
\hline NS1 & 150 & 1258 & 150 & 1176 & 249 & 98 & 85 \\
\hline NEP & 61 & 407 & 54 & 299 & 124 & 31 & 26 \\
\hline NP & 125 & 839 & 93 & 773 & 506 & 61 & 69 \\
\hline M1 & 45 & 467 & 42 & 355 & 275 & 18 & 15 \\
\hline $\mathrm{M} 2$ & 65 & 461 & 64 & 503 & 98 & 25 & 23 \\
\hline PA & 192 & 1677 & 143 & 1358 & 726 & 65 & 47 \\
\hline PA-X & 57 & 164 & 45 & 244 & 252 & 28 & 24 \\
\hline PB1 & 171 & 1654 & 132 & 1347 & 762 & 59 & 33 \\
\hline PB2 & 184 & 1817 & 136 & 1297 & 776 & 52 & 42 \\
\hline PB1-F2 & 151 & 224 & 112 & 737 & 101 & 64 & 54 \\
\hline
\end{tabular}

Total Features are the total number of aa positions that are investigated. Features after MCFS are the aa positions that are ranked significant, i.e. having power to discriminate avian from human sequences

subsets was used to build a classifier, consisting of IFTHEN rules, whose performance was assessed by a 10fold cross-validation (Table 2). HA classifiers for H1N1 and non-structural protein 1 (NS1) classifiers for H3N2 subtypes were the best ones with a mean accuracy of 98 and $98.9 \%$, respectively. Nuclear export protein (NEP) classifiers of the H1N1 subtype and matrix protein 1 (M1) classifiers of the H3N2 subtype had lowest mean accuracy of 83.4 and $88.8 \%$, respectively.

For each protein of each subtype a single rule-based model containing only the most significant rules from their respective 100 classifiers was inferred (Methods). We then reclassified the training data of each protein

Table 2 10-fold cross-validation accuracies

\begin{tabular}{lcc}
\hline & \multicolumn{2}{c}{ Mean accuracy (\%) } \\
\cline { 2 - 3 } Protein & H1N1 & H3N2 \\
\hline HA & 98 & 98.7 \\
M1 & 87.7 & 88.8 \\
M2 & 87.6 & 92.9 \\
NA & 93.9 & 98.6 \\
NP & 93 & 97.3 \\
NS1 & 93.1 & 98.9 \\
NEP & 83.4 & 95.3 \\
PA & 95.1 & 97.9 \\
PA-X & 95.9 & 97.7 \\
PB1 & 94.7 & 95.1 \\
PB1F2 & 95.5 & 92.3 \\
PB2 & 95.9 & 97.5 \\
\hline Cross-valdtion accura
\end{tabular}

Cross-validation accuracies of the 100 classifiers were averaged with its respective rule-based model to get an idea of its performance in terms of classification of human and avian sequences. Polymerase acidic protein $\mathrm{X}$ (PA-X), which is a frame-shift product of the third RNA segment, HA and NEP (NS2) models performed the best (Matthews correlation coefficient $(\mathrm{MCC})=1, \mathrm{MCC}=$ 0.993 , MCC $=0.988$, respectively) among the H3N2 models while HA, NA and NS1 models performed the best among the H1N1 models $(\mathrm{MCC}=0.961, \mathrm{MCC}=$ $0.95, \mathrm{MCC}=0.954$, respectively) (Table 3 ). The poorest of the H1N1 models was the PA-X protein model $(\mathrm{MCC}=0.856)$ and of the H3N2 models was the polymerase basic protein F2 (PB1-F2) protein model $(\mathrm{MCC}=0.861)$. The complete HA H1N1 rule-based model is shown in Table 4. Models for the remaining proteins for both subtypes are provided as supplementary material (Additional file 2).

To further verify the validity of the rule-based models created, we tested them on new, unseen data. This data was protein sequences published at the NCBI resource between 30th of November 2014 and 16th of April 2015. For the H1N1 subtype, the rule-based models of M1, nucleoprotein (NP), NS1, NEP (also called nonstructural protein 2 (NS2)), PB1-F2, polymerase basic protein 1 (PB1) and polymerase basic protein 2 (PB2) provided perfect classification (i.e. all the sequences were correctly classified). For the H3N2 subtype data, the models of HA, M1, NP, NS1, NEP (NS2), polymerase acidic protein (PA), PB1 and PB2 also gave a perfect classification. Table 5 shows the performance of all rule-based models on the unseen data. A list of names of the viruses that could not be classified or were missclassified for both subtypes is given in Additional file 3. 
Table 3 Performance of the models on their corresponding complete data sets

\begin{tabular}{|c|c|c|c|c|c|c|}
\hline \multirow[b]{2}{*}{ Protein } & \multicolumn{3}{|l|}{ H1N1 } & \multicolumn{3}{|l|}{ H3N2 } \\
\hline & Sensitivity & Specificity & $\mathrm{MCC}$ & Sensitivity & Specificity & MCC \\
\hline HA & 0.999 & 0.953 & 0.961 & 1 & 0.987 & 0.993 \\
\hline M1 & 1 & 0.881 & 0.934 & 0.994 & 1 & 0.971 \\
\hline M2 & 1 & 0.859 & 0.918 & 0.996 & 0.873 & 0.908 \\
\hline NA & 1 & 0.907 & 0.95 & 1 & 0.908 & 0.95 \\
\hline NP & 1 & 0.864 & 0.92 & 0.994 & 0.957 & 0.946 \\
\hline NS1 & 0.998 & 0.932 & 0.954 & 0.991 & 0.993 & 0.96 \\
\hline NEP & 0.995 & 0.883 & 0.912 & 0.997 & 1 & 0.988 \\
\hline PA-X & 0.901 & 1 & 0.856 & 1 & 1 & 1 \\
\hline PA & 0.972 & 0.979 & 0.892 & 0.996 & 0.979 & 0.969 \\
\hline PB1-F2 & 0.91 & 0.987 & 0.884 & 0.999 & 0.778 & 0.861 \\
\hline PB1 & 0.993 & 0.93 & 0.923 & 1 & 0.879 & 0.932 \\
\hline PB2 & 0.989 & 0.984 & 0.935 & 0.996 & 0.985 & 0.972 \\
\hline
\end{tabular}

Sensitivity is the ability to correctly predict human sequences and specificity is the ability to correctly predict avian sequences where 1 means perfect prediction and 0 means no correct prediction. Matthews correlation coefficient (MCC) value is a measure of how well the model performs overall where 1 means a perfect classification, 0 is for a prediction no better than random and -1 indicates a total disagreement between predictions and observations. "na" means the measure could not be calculated for the given model

Table 4 Example rule-based model

\begin{tabular}{|c|c|c|c|}
\hline Rule & Accuracy (\%) & Support & $\begin{array}{l}\text { Decision } \\
\text { coverage (\%) }\end{array}$ \\
\hline IF P435 = I THEN host $=$ Human & 99.9 & 5128 & 98.4 \\
\hline IF P200 $=$ S THEN host $=$ Human & 99.9 & 4052 & 77.8 \\
\hline IF P10 = Y THEN host $=$ Human & 99.8 & 3998 & 76.7 \\
\hline IF P88 = S THEN host $=$ Human & 99.9 & 3989 & 76.5 \\
\hline IF P6 $=$ V THEN host $=$ Human & 99.8 & 3936 & 75.5 \\
\hline IF P222 = R THEN host $=$ Human & 99.9 & 3823 & 73.4 \\
\hline IF P220 $=$ T THEN host $=$ Human & 100.0 & 3584 & 68.8 \\
\hline IF P516 = K THEN host $=$ Human & 99.9 & 1818 & 34.9 \\
\hline $\begin{array}{l}\text { IF P200 }=\mathrm{P} \text { and } \mathrm{P} 222=\mathrm{K} \text { THEN } \\
\text { host }=\text { Avian }\end{array}$ & 91.3 & 229 & 97.7 \\
\hline IF P130 $=$ K THEN host $=$ Avian & 91.3 & 218 & 93.0 \\
\hline $\begin{array}{l}\text { IF P2 }=E \text { and P222 }=\text { K THEN } \\
\text { host }=\text { Avian }\end{array}$ & 96.2 & 208 & 93.5 \\
\hline $\begin{array}{l}\text { IF P137 }=\mathrm{A} \text { and } \mathrm{P} 544=\mathrm{L} \text { THEN } \\
\text { host }=\text { Avian }\end{array}$ & 96.1 & 205 & 92.1 \\
\hline $\begin{array}{l}\text { IF P78 }=\mathrm{L} \text { and } \mathrm{P} 435=\mathrm{V} \text { THEN } \\
\text { host }=\text { Avian }\end{array}$ & 97.1 & 204 & 92.5 \\
\hline IF P9 $=$ F THEN host $=$ Avian & 98.5 & 204 & 93.9 \\
\hline IF P6 $=\mathrm{F}$ THEN host $=$ Avian & 98.2 & 169 & 77.6 \\
\hline IF P14 = V THEN host $=$ Avian & 99.4 & 165 & 76.6 \\
\hline IF P173 = T THEN host $=$ Avian & 98.7 & 158 & 72.9 \\
\hline
\end{tabular}

The model presented here is for the HA protein of the H1N1 subtypeModels for the other proteins of both the subtypes are listed in Additional file 2

\section{Predicted host-specific signatures}

The rule-based models allowed us to further interpret them and see how they differentiated viral avian from viral human sequences. Each of the models was analyzed separately for HSSs. The constituent rules of a model associated aa residues at specific positions with an avian or human host. The confidence in these associations is shown as the accuracy, support and the decision coverage shown in the rule-based models. For the combinations in our models we also calculated a combinatorial accuracy gain (CAG), which is the percentage points gain in accuracy of the combination as compared to the average of the accuracies of its constituent singular conditions when taken independently.

\section{Combinatorial signatures}

As expected we found aa combinations, i.e. the combinatorial HSSs, in HA, M1, matrix protein 2 (M2), NP, NS1, NEP (NS2), PA, PA-X, PB1 and PB2 proteins to be associated with specific hosts in the H1N1 subtype. In the H3N2 subtype, we found combinations in M1, M2, NEP, PB1 and PB2 proteins. A complete set of the combinatorial HSSs for both subtypes is given in a supplementary file (see Additional file 4: Combinations_from_rules). Ciruvis diagrams [26] for visualization of combinations of interacting amino acids were used to illustrate the cases of three or more combinations in the models of both subtypes associated with the avian hosts (see Figs. 1 and 2).

Residues $14 \mathrm{G}$ of the M2 H1N1 model and $82 \mathrm{~N}$ of the PB2 H3N2 model were the most connected ones interacting with six other aa residues each. Amino acid 
Table 5 Performance of the rule-based models on the new, unseen data

\begin{tabular}{|c|c|c|c|c|c|}
\hline \multirow[b]{2}{*}{ Protein } & \multicolumn{2}{|c|}{ Human sequences } & \multicolumn{2}{|c|}{ Avian sequences } & \multirow[b]{2}{*}{ Accuracy (\%) } \\
\hline & Total & $\begin{array}{l}\text { Correctly } \\
\text { classified }\end{array}$ & Total & $\begin{array}{l}\text { Correctly } \\
\text { classified }\end{array}$ & \\
\hline $\mathrm{HA}-\mathrm{H} 1 \mathrm{~N} 1$ & 108 & 105 & 2 & 2 & 97.3 \\
\hline $\mathrm{HA}-\mathrm{H} 3 \mathrm{~N} 2$ & 73 & 73 & 4 & 4 & 100.0 \\
\hline M1-H1N1 & 25 & 25 & 0 & 0 & 100.0 \\
\hline $\mathrm{M} 1-\mathrm{H} 3 \mathrm{~N} 2$ & 8 & 7 & 0 & 0 & 87.5 \\
\hline $\mathrm{M} 2-\mathrm{H} 1 \mathrm{~N} 1$ & 30 & 26 & 2 & 2 & 87.5 \\
\hline $\mathrm{M} 2-\mathrm{H} 3 \mathrm{~N} 2$ & 22 & 16 & 3 & 3 & 76.0 \\
\hline $\mathrm{NA}-\mathrm{H} 1 \mathrm{~N} 1$ & 33 & 33 & 2 & 1 & 97.1 \\
\hline $\mathrm{NA}-\mathrm{H} 3 \mathrm{~N} 2$ & 46 & 46 & 4 & 3 & 98.0 \\
\hline NP-H1N1 & 13 & 13 & 2 & 2 & 100.0 \\
\hline $\mathrm{NP}-\mathrm{H} 3 \mathrm{~N} 2$ & 8 & 8 & 4 & 4 & 100.0 \\
\hline NS1-H1N1 & 31 & 31 & 2 & 2 & 100.0 \\
\hline $\mathrm{NS} 1-\mathrm{H} 3 \mathrm{~N} 2$ & 19 & 19 & 3 & 3 & 100.0 \\
\hline NEP-H1N1 & 12 & 12 & 2 & 2 & 100.0 \\
\hline $\mathrm{NEP}-\mathrm{H} 3 \mathrm{~N} 2$ & 8 & 8 & 2 & 2 & 100.0 \\
\hline PAX-H1N1 & 18 & 14 & 2 & 2 & 80.0 \\
\hline PAX-H3N2 & 7 & 7 & 0 & 0 & 100.0 \\
\hline PA-H1N1 & 34 & 29 & 2 & 2 & 86.1 \\
\hline PA-H3N2 & 23 & 23 & 4 & 4 & 100.0 \\
\hline PB1F2-H1N1 & 3 & 3 & 2 & 2 & 100.0 \\
\hline PB1F2-H3N2 & 9 & 8 & 4 & 0 & 61.5 \\
\hline PB1-H1N1 & 27 & 27 & 1 & 1 & 100.0 \\
\hline PB1-H3N2 & 20 & 20 & 1 & 1 & 100.0 \\
\hline PB2-H1N1 & 29 & 29 & 2 & 2 & 100.0 \\
\hline PB2-H3N2 & 16 & 16 & 3 & 3 & 100.0 \\
\hline
\end{tabular}

residues having interactions with more than one other residue, in both the subtypes are listed in Table 6 . These strongly interacting residues might be relatively more essential to host adaptation than the less connected ones.

\section{Singular (linear) signatures}

Previous studies [17-23] mostly found the adaptation signatures on the internal proteins and did not look into surface glycoproteins (HA and NA). In contrast, we found singular HSSs on all the proteins of both subtypes, including the HA, NA and the newly discovered PA-X proteins. In total, 189 singular HSSs were found, in both subtypes combined. Out of these, 132 signatures were novel and not reported by the previous studies (Table 7). A complete list of singular signatures is given in the supplementary material (see Additional file 4: singletons_H3N2, singletons_H1N1).
Specific aa changes predicted to be associated with host adaptation

Some of the rules from our models associated different residues at the same aa positions with avian and human hosts. This can be seen as a mutation (aa change) potentially affecting the adaptation of the viral proteins to a specific host. Eight mutations were found for the H1N1 subtype and 10 for the H3N2 one. In the H1N1 subtype, mutations F6V in HA, P46T and L74V in NA, I6M in both NS1 and NEP and L58- in PB1-F2 were novel. In the H3N2 subtype, mutations R78E in HA, A30I, N40Y and I44S in NA, P28L and R57Q in PA and P28L in PA-X were not identified in the previous studies. Table 8 shows all such mutations in both subtypes.

\section{HSSs are not specific to phylogenetic sub-clades within a host}

The support and the decision coverage of the rules showed whether the HSSs identified were specific to sub-clades or were more general i.e. spread out across the sub-clades. The higher decision coverage indicated more generality of the rule. For example, the top five rules for the avian class have the following very high decision coverage: rule1 $-98.5 \%$, rule $2-98.5 \%$, rule3 $97.8 \%$, rule $4-98.5 \%$ and rule $5-97.8 \%$. It follows that the rules are general. To further illustrate this generality, and to show the diversity in our training data set, a phylogenetic analysis was carried out (Additional file 5). Top five rules specifying each host were mapped onto the created phylogenetic trees, separately for each host, for all the proteins of both subtypes.

As an example, consider the avian PB2 H3N2 tree (Fig. 3). 91.4 \% of the sequences are covered by rule 1, 2, 3,4 and 5 , which is illustrated by the violet coloring of the leaves in the tree. Only, $1.4 \%$ of the sequences were not covered by rule4, yet they were covered by rule 1,2 , 3 , and 5, and similarly for the remaining coverage. For the corresponding human tree, the figures are $89.3 \%$ coverage for the top five human rules. One can see that this generality prevails in all other proteins.

\section{Validity of HSSs across $\mathrm{H} 1 \mathrm{~N} 1$ and $\mathrm{H} 3 \mathrm{~N} 2$ subtypes}

To see whether the HSSs identified in the H1N1 subtype could predict hosts of the H3N2 subtype aa sequences and vice versa, we classified H3N2 subtype data with H1N1 models and H1N1 subtype data with H3N2 models. Good classifications meant that the rules (and consequently the HSSs) generated for one subtype were valid for the other one. Bad classifications meant that the rules of one subtype did not hold for the data of the other subtype and hence no cross-subtype signatures validity. Both HA and NA H1N1 models were bad classifiers for the HA and NA of the H3N2 type data, respectively since they failed to distinguish avian 


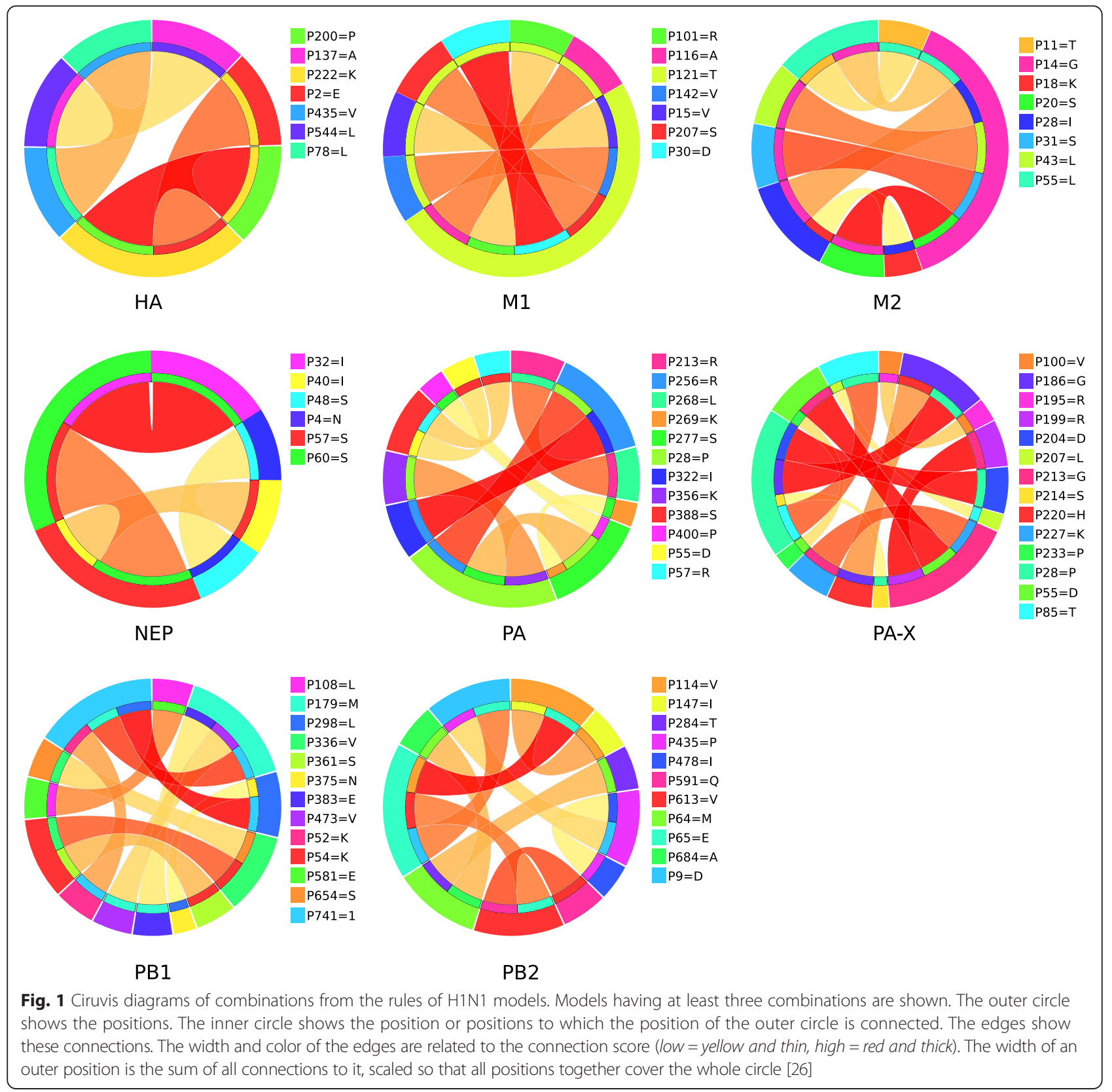

sequences in the data in both cases $(\mathrm{Sp}=0)($ Table 9). It should be kept in mind that the outcome human was considered positive outcome and the outcome avian considered as a negative one. The PA-X H1N1 model could not recognize human sequences in the PA-X H3N2 data $(\mathrm{Sn}=0)$. Furthermore, the models of PA, PB1-F2 and PB1 proteins of H1N1 subtype were bad classifiers of the H3N2 data $(\mathrm{MCC}=-0.11, \mathrm{MCC}=0.056$, MCC $=0.302$ ), specifically failing to identify sequences coming from human hosts $(\mathrm{Sn}=0.021, \mathrm{Sn}=0.023, \mathrm{Sn}=$ 0.563). This meant that H1N1 HSSs in the models of HA, NA, PA-X, PA, PB1-F2 and PB1 proteins were not valid for H3N2 subtype data and these proteins of the H3N2 subtype carried only H3N2-specific HSSs. Contrary to this, the H1N1 models of M1, M2, NP, NS1, NEP and PB2 proteins were able to distinguish between H3N2 subtype sequences coming from avian and human sources reasonably well $(\mathrm{Sn}=0.97-1.0$; $\mathrm{Sp}$ $=0.64-0.94 ; \mathrm{MCC}=0.776-0.941)$. It proved that these proteins of the H3N2 subtype, in addition to the stronger H3N2 HSSs, also carried H1N1 HSSs.

The H3N2 models of HA, NA, NP, NS1, NEP, PA-X and PA proteins could not classify avian and human sequences of $\mathrm{H} 1 \mathrm{~N} 1$ subtype correctly $(\mathrm{MCC}=-0.004-$ 

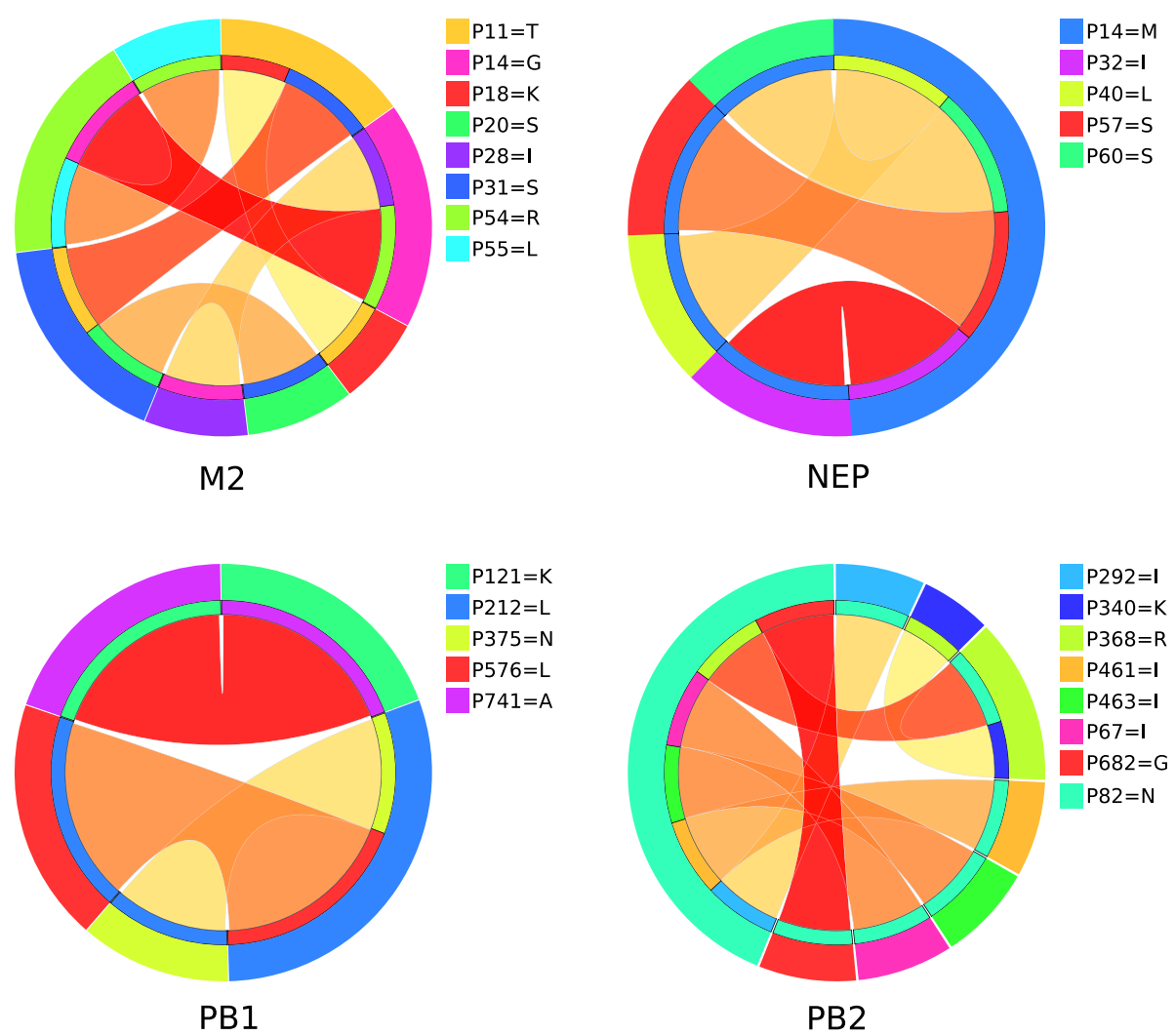

Fig. 2 Ciruvis diagrams of combinations from the rules of H3N2 models. Models having at least three combinations are shown. The outer circle shows the positions. The inner circle shows the position or positions to which the position of the outer circle is connected. The edges show these connections. The width and color of the edges are related to the connection score (low =yellow and thin, high = red and thick). The width of an outer position is the sum of all connections to it, scaled so that all positions together cover the whole circle [26]

Table 6 Amino acid residues having the most interactions in the models of both subtypes

\begin{tabular}{llll}
\hline Subtype & Protein & Positions & Number of interactions \\
\hline H1N1 & HA & $222 \mathrm{~K}$ & 2 \\
& M1 & $121 \mathrm{~T}$ & 5 \\
& M2 & $14 \mathrm{G}$ & 6 \\
& NEP & $57 \mathrm{~S}, 60 \mathrm{~S}$ & 2 \\
& PA & $28 \mathrm{P}, 277 \mathrm{~S}$ & 3 \\
& PA-X & $28 \mathrm{P}$ & 4 \\
& PB1 & $179 \mathrm{M}, 741 \mathrm{~A}$ & 3 \\
& PB2 & $65 \mathrm{E}$ & 3 \\
H3N2 & M1 & $101 \mathrm{R}$ & 2 \\
& M2 & $11 \mathrm{~T}, 14 \mathrm{G}, 31 \mathrm{~S}, 54 \mathrm{R}$ & 2 \\
& NEP & $14 \mathrm{M}$ & 4 \\
& PB1 & $212 \mathrm{~L}$ & 2 \\
& PB2 & $82 \mathrm{~N}$ & 6 \\
\hline
\end{tabular}

0.251). This means that these proteins of the H1N1 subtype carried H1N1-specific HSSs. Whereas the successful classifications of $\mathrm{H} 1 \mathrm{~N} 1$ subtype data of $\mathrm{M} 1$, M2, PB1-F2, PB1 and PB2 proteins by the respective H3N2 models $(\mathrm{MCC}=0.788-0.888 ; \mathrm{Sn}=0.956-0.992$; $\mathrm{Sp}=0.766-0.951)$ proved that these $\mathrm{H} 1 \mathrm{~N} 1$ proteins carried both $\mathrm{H} 1 \mathrm{~N} 1$ and $\mathrm{H} 3 \mathrm{~N} 2$ signatures.

For predicting hosts from an aa sequence we analyzed positions specified by the rules with the remainder of the sequence not taken into account. This meant the existence of a sequence in one or the other phylogenetic clade would not affect the validation of the predicted signatures across subtypes. To prove this point we included sequences of both subtypes into one single phylogeny for all the proteins. In the M1 phylogeny (Fig. 4) the human sequences from both the subtypes formed distinct clades. The avian sequences, on the other hand, did not form separate clades but formed a single clade. This meant that the human sequences were relatively more different between the subtypes than the avian sequences. Across subtype validation of HSSs of the M1 protein proved that the $\mathrm{H} 1 \mathrm{~N} 1$ signatures were valid in 
Table 7 Novel singular aa positions associated to host adaptation

\begin{tabular}{ll}
\hline Protein & Novel singular positions \\
\hline HA & $6,9,10,14,23,47,66,69,78,88,91,94,130,173$, \\
& $189,200,220,222,435,516$ \\
M1 & $30,116,142,207,209$ \\
M2 & $13,16,31,36,43,51,54$ \\
NA & $16,18,19,23,30,40,42,44,46,47,74,79,147,150$, \\
& $157,166,232,285,341,344,351,369,372,389$, \\
& $397,435,437,466$ \\
NP & $31,53,98,146,444,450,498$ \\
NS1 & $6,7,14,23,27,28,74,123,152,192,220,226$ \\
NS2 & $6,7,14,32,34,48,83,86$ \\
PA & $85,323,336,348,362,300$ \\
PAX & $28,85,210,233$ \\
PB1 & $12,54,59,113,175,212,339,435,576,586,587$, \\
& 619,709 \\
PB1F2 & $3,6,12,17,21,25,26,27,28,33,47,52,54,57,58$, \\
& $60,62,65,82$ \\
PB2 & $54,65,354$ \\
\hline
\end{tabular}

H3N2 data, meaning that we could predict the hosts of $\mathrm{H} 3 \mathrm{~N} 2$ human sequences using $\mathrm{H} 1 \mathrm{~N} 1$ signatures. The reason is that the sequences were similar in the analyzed positions. The remainders of the sequence, with comparatively low sequence similarity, did not affect the prediction process. On the other hand, we could also predict the hosts of the H3N2 avian sequences by using H1N1 signatures where the remainders of the sequences had more sequence similarity. And conversely, the H3N2 signatures were also valid for H1N1.

Furthermore, the clades in the NP phylogeny were more or less similar to the M1 phylogeny (Additional file 6). $\mathrm{H} 1 \mathrm{~N} 1$ signatures were valid for $\mathrm{H} 3 \mathrm{~N} 2$ sequences but the converse did not hold, i.e. the H3N2 signatures were not valid for the $\mathrm{H} 1 \mathrm{~N} 1$ sequences.

For the HA and NA phylogeny (Additional file 6), the different subtypes and hosts formed separate clades. The

Table 8 Amino acid changes associated with host adaptation

\begin{tabular}{|c|c|c|c|c|c|c|c|}
\hline \multicolumn{4}{|l|}{ H1N1 } & \multicolumn{4}{|l|}{ H3N2 } \\
\hline Protein & Position & Avian & Human & Protein & Position & Avian & Human \\
\hline $\mathrm{HA}$ & 6 & $\mathrm{~F}$ & V & $\mathrm{HA}$ & 78 & $R$ & $E$ \\
\hline \multirow[t]{2}{*}{ NA } & 46 & $P$ & T & NA & 30 & $A$ & I \\
\hline & 74 & $L$ & V & & 40 & $N$ & Y \\
\hline NP & 100 & R & I,V & & 44 & 1 & S \\
\hline NS1 & 6 & I & M & NP & 16 & G & $D$ \\
\hline NEP & 6 & I & M & PA-X & 28 & P & L \\
\hline PB1-F2 & 58 & L & - & PA & 28 & P & $L$ \\
\hline \multirow[t]{3}{*}{ PB2 } & 588 & A & I & & 57 & R & Q \\
\hline & & & & PB2 & 9 & D & $\mathrm{N}$ \\
\hline & & & & & 64 & M & T \\
\hline
\end{tabular}

cross-subtype validation of signatures failed for these two proteins. However, this failure was not due to the underlying phylogeny; rather the signatures of one subtype could not predict hosts in the other subtype.

It follows that to predict hosts, our method indeed analyzes specific positions in the sequences as specified by the rules, and the remainders of the sequences or the underlying phylogenies do not affect the predictions.

\section{Discussion}

Our models performed reasonably well since all of them had an average accuracy of more than $90 \%$ in the 10 fold cross validation except NEP (NS2), M1 and M2 protein models of the H1N1 type (Accuracy: 83.4, 87.7 and $87.6 \%$, respectively) and M1 protein model of the H3N2 type (Accuracy $88.8 \%$ ) (Table 2). The reason for the somewhat lower accuracies of the above exceptions could be due to either the lack of training sequences from which the models learn or to the absence of stronger HSSs in these sequences.

In previous studies [17-23], signatures of adaptation were mostly found on the internal proteins, especially in viral ribonucleoprotein complexes consisting of viral polymerases and NP. The fact that we were able to build high quality models for all the proteins for both subtypes, indicated that all the proteins, including the highly variable $\mathrm{HA}$ and NA proteins and the recently discovered PA-X protein, carry HSSs. A major difference between our models and the ones previously reported [14-16] is that the previous models were black box classifiers whereas our models are transparent. Black box classifiers give classification but do not provide any straightforward possibility to identify which parameters and for which values a classification is obtained. Transparent classifiers allow explicit analysis of the model, i.e. the features and their values, for each classified object. The models created in this study used aa positions as features and aa residues at those positions as the values for those features, hence lending themselves for easy interpretation and further analysis.

In comparison to previous studies, we identified a larger number of singular HSSs. One reason is that our method requires aa residue at a particular position to be more or less conserved/persistent in one host only. The same position may either have another persistent residue at this position or not have a persistent residue at all. For example, if at a given position in human hosts there is a conserved "Leucine", our method selects this position as a signature of human hosts. The previous studies required that this position be fully or partially conserved in the avian hosts, too, which leads them to having a smaller number of signatures. Furthermore, the previous studies did not analyze the subtypes and the proteins separately. Not limiting analyses to particular 
PB2_H3N2_Avian.tre
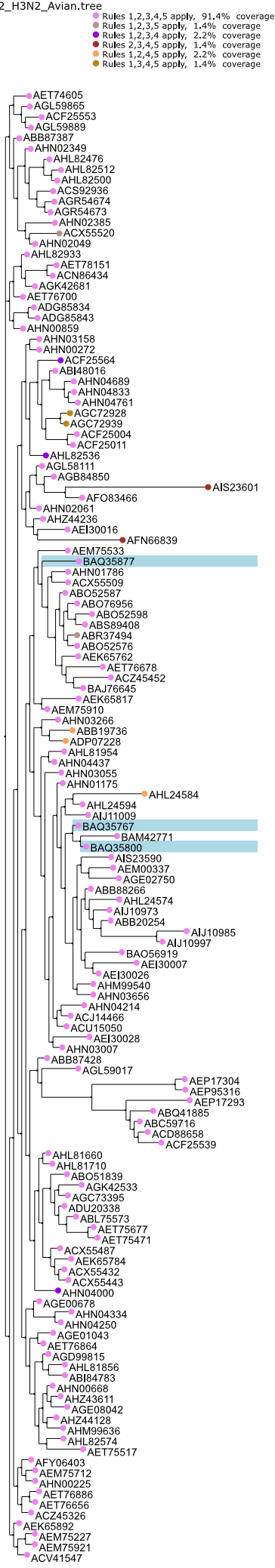

Fig. 3 Phylogeny of PB2 H3N2 protein of avian hosts annotated with top 5 avian rules form the PB2 H3N2 model. Each sequences is represented by its GeneBank accession. The violet nodes mark the sequences that supports rule 1,2,3,4 and 5, which are $91.4 \%$ of the total sequences. Similarly the DarkViolet nodes mark the sequences that support rule 1, 2, 3 and 4 but lacks support for rule 5, which are $2.2 \%$ of the total sequences. The nodes with a LightBlue background are the new, unseen sequences. The unmarked nodes do not support the top 5 rules, and were either supporting rules other than the top 5 or were not classified by the models

subtypes leads to identifying more generic signatures but may loose signatures that are stronger in a subtypespecific manner. Analyzing all the proteins at the same time also results into a smaller number of signatures since the stronger signatures from some proteins may shadow the weaker signatures from the other proteins. We also had more data in some cases. Taubenberger et al.,

Table 9 Performance of the H1N1 models on H3N2 data and vice versa

\begin{tabular}{|c|c|c|c|c|}
\hline & Protein & Sensitivity & Specificity & MCC \\
\hline \multirow[t]{12}{*}{ H3N2 data - H1N1 models } & $\mathrm{HA}$ & 1 & 0 & na \\
\hline & M1 & 1 & 0.895 & 0.941 \\
\hline & M2 & 1 & 0.73 & 0.84 \\
\hline & NA & 1 & 0 & na \\
\hline & NP & 1 & 0.882 & 0.932 \\
\hline & NS1 & 1 & 0.747 & 0.85 \\
\hline & NEP & 1 & 0.648 & 0.78 \\
\hline & PA-X & 0 & 1 & na \\
\hline & PA & 0.021 & 0.93 & -0.11 \\
\hline & PB1-F2 & 0.023 & 1 & 0.056 \\
\hline & PB1 & 0.563 & 0.909 & 0.302 \\
\hline & PB2 & 0.979 & 0.949 & 0.873 \\
\hline \multirow[t]{12}{*}{ H1N1 data - H3N2 models } & $\mathrm{HA}$ & 0 & na & na \\
\hline & M1 & 0.957 & 0.975 & 0.885 \\
\hline & M2 & 0.987 & 0.766 & 0.804 \\
\hline & NA & 1 & 0 & -0.004 \\
\hline & NP & 0.364 & 0.984 & 0.251 \\
\hline & NS1 & 0.365 & 0.993 & 0.237 \\
\hline & NEP & 0.027 & 1 & 0.061 \\
\hline & PA-X & 0.201 & 0.982 & 0.223 \\
\hline & PA & 0.247 & 0.995 & 0.177 \\
\hline & PB1-F2 & 0.991 & 0.804 & 0.832 \\
\hline & PB1 & 0.992 & 0.877 & 0.888 \\
\hline & PB2 & 0.956 & 0.951 & 0.786 \\
\hline
\end{tabular}

Sensitivity is the ability to correctly predict human sequences and specificity is the ability to correctly predict avian sequences where 1 means perfect prediction and 0 means no correct prediction. Matthews correlation coefficient (MCC) value is a measure of how well the model performs overall where 1 means a perfect classification, 0 is for a prediction no better than random and -1 indicates a total disagreement between predictions and observations. "na" means the measure could not be calculated for the given model 


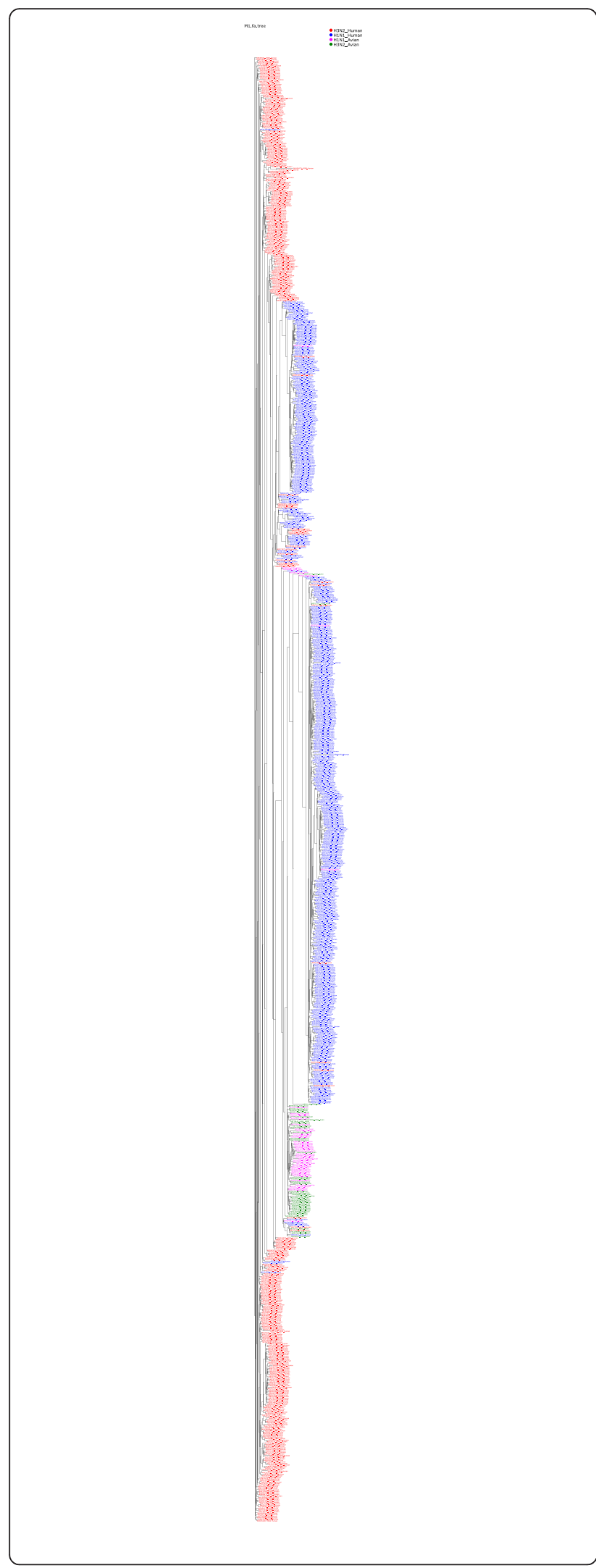

Fig. 4 Phylogenetic tree of the M1 protein from sequences of both subtypes and both hosts. Both the subtypes and the hosts are combined into a single tree. It can be seen that human sequences of both the subtypes form their own distinct clades. The avian sequences, on the other hand, fell into a single clade

[17] had 105, 91 and 83 sequences for proteins PA, PB1 and PB2 while we had 1869,1825 and 2001 in H1N1 and 1501,1479 and 1436 sequences in H3N2 subtype for those proteins respectively. Finkelstein et al., [20] had $\sim 9$ times less data for HA, $\sim 6$ times less for NA and $\sim 4$ times less data for each of the polymerases. Allen et al. [21] had only 281 human sequences and 560 avian sequences for all the proteins.

In addition to singular HSSs, we also identified combinatorial HSSs. Indeed, it is the very first time that combinatorial HSSs are reported in this context. These HSSs are shown as conjunctive rules, i.e., rules with more than one condition in the IF part. It appeared that some aa residues were part of more than one combination in our models. This may suggest that these residues are potentially more important in establishing host adaptation then the ones appearing in one combination only (Table 6).

In the M2 H1N1 model, the combinations associated with avian hosts had a Glycine $(\mathrm{G})$ residue at position 14 while the combinations for human hosts had a Glutamic acid (E) in the same position. Similarly, in PB2 H3N2 model, Arginine (R) at position 340 was associated to avian hosts while Lysine (K) residue at the same position to human hosts. It seems that the mutations G14E in M2 H1N1 and R340K in PB2 H3N2 model potentially facilitate the shift of hosts from avian to human. However, these residues always appear in combination with other residues and therefore they are HSSs only in combinations and not individually. The reason is obvious. The confidence measures (accuracy, support and decision-coverage) were calculated for the combination as a whole. We do not report such mutations in our list of mutations, although they indicate an effect. The functions of these combinations at a molecular level are not understood yet, but they provide a novel and interesting perspective of looking at sequencebased host adaptation.

The method used in this study is also a sequencebased method like phylogeny. Phylogeny puts sequences in clades and sub-clades based on the similarity or difference of the complete sequences but it does not output how exactly or at what positions the sequences are different. Our classification method identifies the exact aa differences (the HSSs) between the sets of sequences. For the sake of an example, let us assume that at a given position the avian viruses carry a conserved Methionine and at the same position in the human viruses 
there is a conserved Alanine. This position will be identified by our method as a host-specific signature. The remainder of the sequence does not affect the identification process. In order to simplify the argument we consider two extreme cases. a) The remainders of the sequences are identical. The two sequences will most likely be put into the same phylogenetic clade. However, our method will select the afore-mentioned position because it differentiates avian and human viruses. b) The remainders of the sequences differ entirely. The sequences will be assigned to different phylogenetic clades, while our method will select the said position since it nonetheless differentiates the viruses. It follows that our method is invariant of the underlying phylogeny. In practice, however, since we are predicting the variable host that is not independent of phylogeny, some of the HSSs discussed may inform on the phylogeny.

HA and NA of both subtypes were found to be only carrying subtype-specific HSSs. This goes well with the current knowledge that these two proteins are the most diverse proteins that are specifically adapted to interact with the host cell. M1, M2 and PB2 are shown to be the most conserved proteins from the point of view of host specifying genomic signatures since they carried the HSSs valid for both subtypes.

The HSSs found in this study were also considered in other contexts in other studies such as viral viability and antiviral resistances. For instance, positions 30, 142, 207 and 209 occurring in the H1N1 M1 models have been previously shown to affect viral production when mutated [27], while mutation S31N derived from M2 models is a known marker of amantadine resistance [28-31]. Table 10 lists all the aa residues and their descriptions as found in different contexts in the literature. All these different contexts, that the aa residues from our models are described in, show that they affect the fitness of the viruses in one or the other way, which in turn facilitates their adaptation to the new environment or hosts.

\section{Conclusions}

The highly predictive rule-based models built for 12 proteins for $\mathrm{H} 1 \mathrm{~N} 1$ and $\mathrm{H} 3 \mathrm{~N} 2$ subtypes suggest that there are HSSs on all the protein including the diverse HA, $\mathrm{NA}$ and the newly discovered PA-X protein that were not previously studied in this context. In addition, the transparent nature of our method allowed us to further investigate our models for how the predictions were actually done. This resulted in lists of predicted singular and combinatorial HSSs. Some of the HSSs identified in this study were already known while others are novel. The ability of our methods to capture combinatorial HSSs that may affect the host adaptation process makes this study unique. We discovered that the surface proteins HA and NA carry subtype-specific HSSs in both subtypes while NP, NS1, NEP, PA-X and PA of the H1N1 subtype and PA-X, PA, PB1-F2 and PB1 of the H3N2 subtype carry subtype-specific HSSs. We showed that M1, M2, PB1-F2, PB1 and PB2 of the H1N1 subtype carried H1N1 and some additional H3N2 HSSs, and vice versa, M1, M2, NP, NS1, NEP and PB2 of the H3N2 subtype carried H3N2 and some additional H1N1 signatures. The computational results presented here will eventually require further analysis by testing the host-pathogen interactions under laboratory conditions. We believe that the computational analyses provide important support in the characterization of host-pathogen interactions and the proper combination of in silico and in vitro (probably even in vivo) studies will yield important novel information concerning the infection biology of various viruses and other infectious agents.

\section{Methods}

The combined feature selection - rule-based modeling methodology used in this is similar to our previous work where we identified a complete map of potential pathogenicity markers in the $\mathrm{H} 5 \mathrm{~N} 1$ subtype of the avian influenza A viruses [32].

\section{Data}

The data used to make the models was downloaded from the NCBI flu database found at http://www.ncbi.nlm.nih.gov/genomes/FLU/Database/nph-select.cgi?go=database [33]. Full-length plus (nearly complete, may only miss the start and stop codons) protein sequences of the twelve proteins namely, HA, NA, NP, M1, M2, NS1, NEP (NS2), PA, PA-X, PB1, PB2 and PB1-F2, were separately downloaded as published up till November 30, 2014. Identical sequences were represented by the oldest sequence in the database. For each protein, sequences of the H3N2 and H1N1 subtypes of avian and human hosts were downloaded. Sequences of the mixed subtypes were not included in this study. Table 1 shows the number of sequences for each of the proteins for each subtype. For each protein we combined the sequences of the two subtypes used in this study into a single file and aligned them with MUSCLE (v3.8.31) [34].

\section{Decision tables}

A decision table was created for each of the proteins for both the subtypes. A decision table can be seen as a tabularized form of the aligned FASTA sequences with an extra decision/label column, which in our case was the host information. The first column of the decision tables contained the identifier of the sequence, and the last column was the label/outcome column, the host information in our case and the rest of the columns represented the sequence information corresponding to the aligned FASTA files. The alignment gaps were represented by a '?' in the decision 
Table 10 Amino acid positions discussed in literature from the models of both the subtypes for all proteins

\begin{tabular}{|c|c|c|}
\hline Protein & Positions & Description \\
\hline \multirow[t]{4}{*}{ M1 } & $115,121,137$ & Known signatures of host-adaptation $[19,22,23]$ \\
\hline & $30,142,207,209$ & Affecting viral production on mutation [27] \\
\hline & 121 & Affecting viral replication [45] \\
\hline & 101 & $\begin{array}{l}\text { Determinant of temperature sensitivity [46], located in a transcription } \\
\text { inhibition site [47] and is also interacting with NEP [48] }\end{array}$ \\
\hline \multirow[t]{3}{*}{ M2 } & $11,14,18,20,28,55,57,78,82,89,93$ & Known signatures of host-adaptation $[19,22,23,49]$ \\
\hline & 31 & $\mathrm{~S} 31 \mathrm{~N}$ is a known marker for amantadine resistance [28-31] \\
\hline & 18,20 & Lie next to 17,19 which forms a di-sulphide bond [50] \\
\hline \multirow[t]{5}{*}{ NS1 } & $18,21,22,53,60,70,81,112,114,171,215,227$ & Known signatures of host-adaptation $[18,20-23,51]$ \\
\hline & 215 & Required for Crk/CrL-SH3 binding [52] \\
\hline & 123 & $\begin{array}{l}\text { Necessary for interaction with PKR, resulting in an inhibition of elF2alpha } \\
\text { phosphorylation [53] }\end{array}$ \\
\hline & 95 & $\begin{array}{l}\text { Along with others, has been shown to be necessary for } \\
\text { binding p85beta and activating PI3K signaling }[54,55]\end{array}$ \\
\hline & 220 & $\begin{array}{l}\text { Part of nuclear localization signal } 2 \text { essential for the } \\
\text { importin-alpha binding [56] }\end{array}$ \\
\hline NEP(NS2) & $57,60,70,107$ & Known signatures of host-adaptation $[18,19,22,23,57]$ \\
\hline \multirow[t]{2}{*}{ NP } & $16,33,100,214,283,313,351,353,357,422$ & Known signatures of host-adaptation $[19-23,58]$ \\
\hline & 16 & D16G shown to decrease pathogenicity several fold [59] \\
\hline \multirow[t]{3}{*}{ PA } & $28,55,57,65,256,268,277,356,382,400,409$ & Known signatures of host-adaptation $[19-23,58]$ \\
\hline & 85,336 & $\begin{array}{l}\text { Residues } 85 \mathrm{I} \text { and } 336 \mathrm{M} \text { are deemed important for enhanced } \\
\text { polymerase activity in mammalian cells [60] }\end{array}$ \\
\hline & $57,65,85$ & $\begin{array}{l}\text { Shown to be involved in suppressing the host cell protein synthesis } \\
\text { during infection [61] }\end{array}$ \\
\hline \multirow[t]{3}{*}{ PB1 } & $52,179,216,298,327,336,361,375,581,741$ & Known signatures of host-adaptation $[17,19,22,23,58]$ \\
\hline & 581 & $\begin{array}{l}\text { Shown to be conferring temperature sensitivity to human influenza } \\
\text { virus vaccine strains [62] }\end{array}$ \\
\hline & 473 & $\begin{array}{l}\text { Mutation at position } 473 \text { has been shown to decrease polymerase } \\
\text { activity [63] }\end{array}$ \\
\hline \multirow[t]{4}{*}{ PB2 } & $9,44,64,81,105,271,292,368,453,588,613,682,684$ & Known signatures of host-adaptation $[19,20,22,23,58]$ \\
\hline & 591 & $591 \mathrm{Q}$ is known to mimic the effect of $627 \mathrm{~K}[64,65]$ \\
\hline & 271 & 271A shown to increase polymerase activity in mammalian cells [66] \\
\hline & 271,588 & Also been shown to be host range determinants [67] \\
\hline \multirow[t]{2}{*}{ PB1-F2 } & $16,23,42,66,70,73,76$ & Known signatures of host-adaptation $[18,23]$ \\
\hline & 66 & Linked with affecting pathogenicity [68] \\
\hline \multirow[t]{2}{*}{ NA } & $46,47,74,147,157,341,351$ & Under selection pressure with a shift of hosts from birds to humans [58] \\
\hline & 344 & Calcium ion binds here that stabilizes the molecule (UniProt: Q9IGQ6). \\
\hline \multirow[t]{2}{*}{ HA } & $2,6,9,10,14$ & Signal peptide domain \\
\hline & $88,173,220,22$ & $\begin{array}{l}\text { Position 71, 159, } 206 \text { and } 208 \text { of the fully-mature } \mathrm{HA} \text { with } \\
\mathrm{H3} \text {-numbering [69]) are part of the antigenic sites } \mathrm{Cb} \text {, Sb } \\
\text { and Ca of the HA protein, respectively [70, 71] }\end{array}$ \\
\hline
\end{tabular}

tables. The rows of a decision table were called objects each representing a particular aa sequence and a label. Columns other than the first and the last one were the features.

\section{Feature selection}

MCFS, as described in [25], was used to rank the features of the decision tables with respect to their ability to discern between avian and human hosts. MCFS is implemented as a software package dmLab [35]. MCFS uses a large number of decision trees and assigns a normalized relative importance (RI-norm) score to each feature such that the features contributing more to the discernibility of the outcome gets a higher score. Statistical significance of the RI-norm scores was assessed with a permutation test 
and significant features $(p<0.05)$, after Bonferroni correction [36], were kept as described in [37]. Only these features were used in the further rule-based model generation.

\section{Under-sampling the data sets}

In the training data for both subtypes, the number of sequences from human hosts was considerably higher than that from the avian hosts. It has previously been shown that this imbalance affects the learning in favor of the dominating class [38]. However to address this problem one can artificially balance the classes [39]. To this end, a technique called under-sampling was used where the sequences belonging to the dominating class were randomly sampled equal to the class having the lesser number of sequences and repeated this step 100 times. In this way for each protein and for each subtype we created 100 subsets where the number of sequences belonging to human and avian hosts were equal. A single rule-based classifier was inferred from each of the subsets, which resulted in 200 classifiers per protein (100 for each subtype). We illustrate the process with the following example.

The data set of the NA protein of the H1N1 subtype had 3093 human and 205 avian sequences, which was a significant imbalance in the number of sequences. From the human set we created subsets by randomly extracting 100 times 205 human sequences and joining them with the 205 avian sequences to create 100 subsets.

\section{Rough sets and rule-based model generation}

Rough set theory [24] was used to produce minimal sets of features that can discern between the objects belonging to different decision classes. ROSETTA [40], a publicly available software system that implements rough sets theory, was used to transform the minimal sets of features into rule-based models [41] that consisted of simple IF-THEN rules. A complete description of rough sets can be found in [42] and the combined MCFS-ROSETTA approach to model generation in bioinformatics is described in [43].

The input data to ROSETTA were the balanced decision tables created in the previous step with only the significant features obtained from applying MCFS. ROSETTA computed approximately minimal subsets of feature combinations that discerned between avian and human hosts with the Johnsons algorithm implemented in ROSETTA. The classifiers were collections of IFTHEN rules. A sample rule from the HA-H1N1 model:

\begin{tabular}{llll}
\hline Rule & Accuracy (\%) & Support & $\begin{array}{l}\text { Decision } \\
\text { coverage (\%) }\end{array}$ \\
\hline $\begin{array}{l}\text { IF P200 = P AND P222 =K } \\
\text { THEN host = Avian }\end{array}$ & 91.3 & 229 & 97.7 \\
\hline
\end{tabular}

reads as: "IF at position 200 there is a Proline residue AND at position 222 there is a Lysine residue THEN the sequence is from an avian host".

There is additional information about the rules available. Support is the set of sequences (229 sequences) that satisfy the conditions of the left hand side (LHS), i.e. the set of sequences that have a proline residue at position 200 and a lysine residue at position 222. For this rule, Accuracy is $91.3 \%$ that is the proportion of correctly classified sequences to the total number of supporting sequences (209/229). Human sequences are considered positive and avian as negatives in this study. The decision coverage for this rule is $97.7 \%$, which means it correctly classifies $97.7 \%$ of the total avian sequences used to train the classifier. It is calculated as follows:

$$
\text { Decision Coverage }(\%)=\left(\frac{\text { Accuracy } \times \text { Support }}{\text { Total training objects of the decision class }}\right) \times 100
$$

Accuracy $\times$ Support gives us the total number of sequences that are correctly classified by the rule. Since the rule is for the avian decision class, the total number of avian sequences used to train the classifier was 214 . So for the stated rule the decision coverage will be $((0.913 * 229) / 214) * 100$, which is equal to $97.7 \%$. The above rule is a conjunctive rule since there is a conjunction of conditions $(\mathrm{P} 200=\mathrm{P}$ AND P222 $=\mathrm{K})$ in the left hand side (LHS) of the rule. A conjunctive rule captures the combinatorial HSSs. Each conjunctive rule must always be used as combination only, because the support, accuracy and the decision coverage measures are calculated for the conjunction and not for the individual conjuncts. A rule can also be a singleton rule where LHS consists of only a single condition.

The confidence in these classifiers come from the 10fold cross validation performed in ROSETTA. In a 10fold cross validation step the input data set is randomly divided into ten equal subsets, say $\{\mathrm{P} 1, \ldots, \mathrm{P} 10\}$. A classifier is trained on the first nine subsets $\{\mathrm{P} 1, \ldots, \mathrm{P} 9\}$ and then tested on the remaining, P10 subset. In the next run, another classifier is trained on $\{\mathrm{P} 1, \ldots, \mathrm{P} 8, \mathrm{P} 10\}$ and its performance is tested on the remaining subset, this time P9. Notice that each time the test set is a different one. The process is repeated 10 times and by then each subset has been used once as a test set. The performance of all the classifiers is averaged and presented as a crossvalidation accuracy. Such a validation is quite common in machine learning since one becomes more or less assured that the performance of the classifier was not simply by chance.

\section{Extraction of a single rule-based model for each protein}

Rules from all the 100 classifiers were combined into a single file. Duplicates were removed. Among partially 
identical rules, the one with the highest decision coverage was kept. If the difference of decision coverage was lower than $1 \%$ then the shortest (the rule with least conditions) was kept. Accuracy, support and decision coverage were calculated on the complete data set for all the rules. Rules that were below the $90 \%$ accuracy and $30 \%$ decision coverage thresholds were discarded. In this way we extracted a single, high quality rule-based model for each of the protein for both H1N1 and H3N2 subtype data.

\section{Classification of sequences}

In order to classify a sequence, each rule from the model was applied on it. If the conditions of the rule matched the sequence, the rule was said to fire on the sequence. Every fired rule voted for a particular classification specified by its THEN-part. The number of votes a fired rule casted was the accuracy multiplied by the support of the rule. For a sequence several rules may fire, each casting votes in favor of the class in the THEN-part. The final classification was assigned based on the majority of votes.

Consider the rules:

1) IF $P 70=S$ THEN host $=$ Avian. Acc $=94.0 \%$. Supp $=50$

2) IF P14 $=\mathrm{M}$ and $\mathrm{P} 32=\mathrm{I}$ THEN host $=$ Avian. Acc $=93.0 \%$. Supp $=43$

3) IF P14 = L THEN host $=$ Human. Acc $=100 \%$. Supp $=285$

4) IF P57 $=$ L THEN host $=$ Human. Acc $=100 \%$. Supp $=273$

Now let us assume that these four rules are applied to a sequence an it turns out that Rule 2, 3 and 4 fire for this sequence. Rule 2 will cast $40(0.93 * 43)$ votes for class Avian while rule 2 and rule 3 will cast 285 and 273 votes in favor of class Human. So, the sequence will be classified as class Human since the number of votes is 558 versus 40 .

In case of no rules fired or there was a tie in the votes, the sequences were labeled as unknown.

\section{Performance evaluation statistics of the rule-based models}

In this study the outcome human was considered as a positive outcome and outcome avian was considered as a negative one. True positives (TP) were sequences correctly classified as coming from human hosts. True negatives (TN) were sequences correctly classified as coming from avian hosts. False positives (FP) were actually avian sequences but incorrectly classified as human sequences and false negatives (FN) were actually human sequences that were incorrectly classified as avian sequences. The performance of the models for all the proteins for both $\mathrm{H} 1 \mathrm{~N} 1$ and $\mathrm{H} 3 \mathrm{~N} 2$ was assessed by the following statistics.

Sensitivity: it is also known as the true positive rate (TPR). In our case, rate at which a model correctly identifies sequences coming from a human host is the sensitivity i.e. a sequence originally from human host and classified as coming from human hosts by the model. It is calculated with the following formula:

$$
\text { Sensitivity }(S n)=\frac{T P}{(T P+F N)}
$$

Specificity: Also known as the true negative rate (TNR). The rate at which the model correctly identifies avian sequences is the specificity, which is calculated by:

$$
\text { Specificity }(S p)=\frac{T N}{(F P+T N)}
$$

Matthews correlation coefficient: It is a measure of how well a model classifies as a whole. The difference with accuracy is that unlike accuracy Matthews correlation coefficient is not affected by un-balanced data and hence gives a better overall idea of how well the model is classifying. It is calculated by the following formula:

$$
\begin{aligned}
& \text { Matthews correlation coefficient }(M C C) \\
& =\frac{(T P \times T N)-(F P \times F N)}{\sqrt{(T P+F P) \times(T P+F N) \times(T N+F P) \times(T N+F N)}}
\end{aligned}
$$

\section{From alignment positions to true positions}

In this study the aa positions for all the $\mathrm{H} 3 \mathrm{~N} 2$ proteins except the PB1-F2 corresponds to the positions of the A/Victoria/JY2/1968 virus. For all but PB1-F2 proteins of the H1N1 data, the positions shown in this study correspond to positions on the A/Wisconsin/301/1976 virus. The PB1-F2 protein for both viruses is in a truncated form and we wanted to show positions from a fulllength protein. For this reason we mapped the PB1-F2 H3N2 positions to the PB1-F2 of the A/New York/674/ 1995 virus and the PB1-F2 H1N1 positions to full-length PB1-F2 of the $A /$ duck/Korea/372/2009 virus.

\section{Phylogenetic analysis}

FastTree 2.1.8 [44] was used to create the phylogeny trees.

\section{Scripting programming language}

Python was used for scripting purposes.

\section{Additional files}

Additional file 1: This file contains the lists of significant features that were selected by MCFS for all the proteins of both subtypes. (XLSX 74 kb) 
Additional file 2: This file contains the rule-based models for all the proteins of both subtypes. (XLSX $41 \mathrm{~kb}$ )

Additional file 3: This file contains list of names of the unseen viral sequences for both subtypes that were either miss-classified or could not be classified by the rule-based models. (XLSX $10 \mathrm{~kb}$ )

Additional file 4: This file contains singular and combinatorial signatures from the rules for both subtypes. (XLSX 63 kb)

Additional file 5: This file contains all the phylogeny trees, separate for subtype and host, marked with top 5 rules. Each sequence is represented by its GeneBank accession. The nodes with a LightBlue background are the new, unseen sequences. The unmarked nodes do not support the top 5 rules, and were either supporting rules other than the top 5 or were not classified by the models. (PDF $7180 \mathrm{~kb}$ )

Additional file 6: This file contains all the combined subtypes and hosts phylogeny trees for each protein. Each sequence is represented by its GeneBank accession, its subtype and its host. (PDF 8113 kb)

\section{Abbreviations}

aa, amino acids; CAG, combinatorial accuracy gain; HA, hemagglutinin; HSSs, host-specific signatures; IAVs, influenza A viruses; LHS, left hand side; M1, matrix protein 1; M2, matrix protein 2; MCC, Matthews correlation coefficient; MCFS, Monte carlo feature selection; NA, neuraminidase; NEP, nuclear export protein; NP, nucleoprotein; NS1, non structural protein 1; NS2, non structural protein 2; PA, polymerase acidic protein; PB1, polymerase basic protein 1; PB2, polymerase basic protein 2; Sn, sensitivity; Sp, specificity

\section{Acknowledgements}

We would like to thank Husen Umer who gave valuable comments during various stages of the work.

\section{Funding}

This research was supported by Uppsala University, Sweden, the ESSENCE grant, (ZK and JK), JK was supported in part by Institute of Computer Science, Polish Academy of Sciences, Poland. The EMIDA ERA-NET FP7 EU projects Epi-SEQ (nr. 219235), NADIV (nr. ID 108), the SLU Award of Excellence provided support to SB, and the Swedish Research Council FORMAS Strong Research Environments project, nr 2011-1692, "BioBridges") to ML and SB.

\section{Availability of data and materials}

The datasets (multiple alignments and phylogenies) supporting the conclusions of this article are available in the DRYAD repository, http://dx.doi.org/10.5061/ dryad.kc097.

\section{Authors' contributions}

ZK has performed all computational experiments. Together with JK they were the main contributors to the paper. MK and SB have contributed the idea to analyze the virus data following the earlier work of JK. They also contributed to writing the paper. JK provided the computational methods and supervised the work. All the authors have read and approved the manuscript.

\section{Competing interests}

The authors declare that they have no competing interests.

\section{Consent for publication}

Not applicable.

\section{Ethics approval and consent to participate}

\section{Not applicable.}

\section{Author details}

${ }^{1}$ Department of Cell and Molecular Biology, Computational Biology and Bioinformatics, Science for Life Laboratory, Uppsala University, SE-751 24 Uppsala, Sweden. ${ }^{2}$ Department of Virology, Parasitology and Immunobiology (VIP), National Veterinary Institute (SVA), Uppsala, Sweden. ${ }^{3}$ OIE Collaborating Centre for the Biotechnology-based Diagnosis of Infectious Diseases in Veterinary Medicine, Ulls väg 2B and 26, SE-756 89 Uppsala, Sweden. ${ }^{4}$ Department of Biomedical Sciences and Veterinary Public Health (BVF), Swedish University of Agricultural Sciences (SLU), Uppsala, Sweden. ${ }^{5}$ Institute of Computer Science, Polish Academy of Sciences, 01-248 Warszawa, Poland.
Received: 30 September 2015 Accepted: 7 July 2016

Published online: 29 July 2016

\section{References}

1. Shi Y, Wu Y, Zhang W, Qi J, Gao GF. Enabling the 'host jump': structural determinants of receptor-binding specificity in influenza A viruses. Nat Rev Microbiol. 2014;12(12):822-31. doi:10.1038/nrmicro3362.

2. Steel J, Lowen AC. Influenza A virus reassortment. Curr Top Microbiol Immunol. 2014;385:377-401. doi:10.1007/82 2014 395.

3. cdc. Influenza (Seasonal) Fact Sheet. 2014. http://www.who.int/mediacentre/ factsheets/fs211/en/. Accessed 17 April 2015.

4. Taubenberger JK, Morens DM. Pandemic influenza-including a risk assessment of H5N1. Rev Sci Tech. 2009;28(1):187-202.

5. Kilbourne ED. Influenza pandemics of the 20th century. Emerg Infect Dis. 2006;12(1):9-14. doi:10.3201/eid1201.051254.

6. Gamblin SJ, Skehel JJ. Influenza hemagglutinin and neuraminidase membrane glycoproteins. J Biol Chem. 2010;285(37):28403-9. doi:10.1074/ jbc.R110.129809.

7. Tong S, Li Y, Rivailler P, Conrardy C, Castillo DA, Chen LM, et al. A distinct lineage of influenza A virus from bats. Proc Natl Acad Sci U S A. 2012; 109(11):4269-74. doi:10.1073/pnas.1116200109.

8. Tong S, Zhu X, Li Y, Shi M, Zhang J, Bourgeois M, et al. New world bats harbor diverse influenza A viruses. PLoS Pathog. 2013;9(10):e1003657. doi:10. 1371/journal.ppat.1003657.

9. Reid AH, Fanning TG, Hultin JV, Taubenberger JK. Origin and evolution of the 1918 "Spanish" influenza virus hemagglutinin gene. Proc Natl Acad Sci U S A. 1999;96(4):1651-6.

10. Garten RJ, Davis CT, Russell CA, Shu B, Lindstrom S, Balish A, et al. Antigenic and genetic characteristics of swine-origin 2009 A(H1N1) influenza viruses circulating in humans. Science. 2009:325(5937):197-201. doi:10.1126/science.1176225.

11. Matrosovich MN, Gambaryan AS, Teneberg S, Piskarev VE, Yamnikova SS, Lvov DK, et al. Avian influenza A viruses differ from human viruses by recognition of sialyloligosaccharides and gangliosides and by a higher conservation of the HA receptor-binding site. Virology. 1997;233(1):224-34. doi:10.1006/viro.1997.8580

12. Li OT, Chan MC, Leung CS, Chan RW, Guan Y, Nicholls JM, et al. Full factorial analysis of mammalian and avian influenza polymerase subunits suggests a role of an efficient polymerase for virus adaptation. PLoS One. 2009;4(5): e5658. doi:10.1371/journal.pone.0005658.

13. Subbarao EK, London W, Murphy BR. A single amino acid in the PB2 gene of influenza A virus is a determinant of host range. J Virol. 1993:67(4):1761-4.

14. Qiang $X$, Kou Z. Prediction of interspecies transmission for avian influenza A virus based on a back-propagation neural network. Math Comput Model. 2010;52(11-12):2060-5. http://dx.doi.org/10.1016/j.mcm.2010.06.008.

15. Wang J, Ma C, Kou Z, Zhou YH, Liu HL. Predicting transmission of avian influenza A viruses from avian to human by using informative physicochemical properties. Int J Data Min Bioinform. 2013;7(2):166-79.

16. Eng $\mathrm{CL}$, Tong JC, Tan TW. Predicting host tropism of influenza A virus proteins using random forest. BMC Med Genomics. 2014;7 Suppl 3:S1. doi:10.1186/1755-8794-7-S3-S1.

17. Taubenberger JK, Reid AH, Lourens RM, Wang R, Jin G, Fanning TG. Characterization of the 1918 influenza virus polymerase genes. Nature. 2005; 437(7060):889-93. doi:10.1038/nature04230.

18. Chen GW, Chang SC, Mok CK, Lo YL, Kung YN, Huang JH, et al. Genomic signatures of human versus avian influenza A viruses. Emerg Infect Dis. 2006;12(9):1353-60. doi:10.3201/eid1209.060276.

19. Chen GW. Shih SR. Genomic signatures of influenza A pandemic (H1N1) 2009 virus. Emerg Infect Dis. 2009;15(12):1897-903. doi:10.3201/eid1512.090845.

20. Finkelstein DB, Mukatira S, Mehta PK, Obenauer JC, Su X, Webster RG, et al. Persistent host markers in pandemic and H5N1 influenza viruses. J Virol. 2007:81(19):10292-9. doi:10.1128/JVl.00921-07.

21. Allen JE, Gardner SN, Vitalis EA, Slezak TR. Conserved amino acid markers from past influenza pandemic strains. BMC Microbiol. 2009;9:77. doi:10.1186/ 1471-2180-9-77.

22. Miotto O, Heiny AT, Albrecht R, Garcia-Sastre A, Tan TW, August JT, et al. Complete-proteome mapping of human influenza A adaptive mutations: implications for human transmissibility of zoonotic strains. PLoS One. 2010; 5(2):e9025. doi:10.1371/journal.pone.0009025.

23. Hu YJ, Tu PC, Lin CS, Guo ST. Identification and chronological analysis of genomic signatures in influenza A viruses. PLoS One. 2014;9(1):e84638. doi:10.1371/journal.pone.0084638. 
24. Pawlak Z. Rough sets. Int J Comput Inform Sci. 1982;11(5):341-56. doi:10.1007/BF01001956.

25. Draminski M, Rada-Iglesias A, Enroth S, Wadelius C, Koronacki J, Komorowski J. Monte Carlo feature selection for supervised classification. Bioinformatics. 2008;24(1):110-7. doi:10.1093/bioinformatics/btm486.

26. Bornelov S, Marillet S, Komorowski J. Ciruvis: a web-based tool for rule networks and interaction detection using rule-based classifiers. BMC Bioinformatics. 2014;15:139. doi:10.1186/1471-2105-15-139.

27. Bialas KM, Desmet EA, Takimoto T. Specific residues in the 2009 H1N1 swine-origin influenza matrix protein influence virion morphology and efficiency of viral spread in vitro. PLoS One. 2012;7(11):e50595. doi:10.1371/ journal.pone.0050595.

28. Abed Y, Goyette N, Boivin G. Generation and characterization of recombinant influenza $A(H 1 N 1)$ viruses harboring amantadine resistance mutations. Antimicrob Agents Chemother. 2005;49(2):556-9. doi:10.1128/ AAC.49.2.556-559.2005

29. He G, Qiao J, Dong C, He C, Zhao L, Tian Y. Amantadine-resistance among H5N1 avian influenza viruses isolated in Northern China. Antiviral Res. 2008; 77(1):72-6. doi:10.1016/j.antiviral.2007.08.007.

30. Cheung CL, Rayner JM, Smith GJ, Wang P, Naipospos TS, Zhang J, et al. Distribution of amantadine-resistant $\mathrm{H} 5 \mathrm{~N} 1$ avian influenza variants in Asia. J Infect Dis. 2006;193(12):1626-9. doi:10.1086/504723.

31. Ilyushina NA, Govorkova EA, Webster RG. Detection of amantadine-resistant variants among avian influenza viruses isolated in North America and Asia. Virology. 2005;341 (1):102-6. doi:10.1016/j.virol.2005.07.003.

32. Khaliq Z, Leijon M, Belak S, Komorowski J. A complete map of potential pathogenicity markers of avian influenza virus subtype $\mathrm{H} 5$ predicted from 11 expressed proteins. BMC Microbiol. 2015;15:128. do:10.1186/s12866-015-0465-x.

33. Bao Y, Bolotov P, Dernovoy D, Kiryutin B, Zaslavsky L, Tatusova T, et al. The influenza virus resource at the National Center for Biotechnology Information. J Virol. 2008;82(2):596-601. doi:10.1128/jvi.02005-07.

34. Edgar R. MUSCLE: multiple sequence alignment with high accuracy and high throughput. Nucleic Acids Res. 2004;32(5):1792-7.

35. Draminski M. Michal Dramiski home page. 2014. http://www.ipipan.waw. $\mathrm{pl} / \sim \mathrm{mdramins} / \mathrm{software.htm}$. Accessed 10 Dec 2014.

36. Holm S. A simple sequentially rejective multiple test procedure. Scandinavian journal of statistics. 1979;6:65-70.

37. Bornelov S, Saaf A, Melen E, Bergstrom A, Torabi Moghadam B, Pulkkinen V, et al. Rule-based models of the interplay between genetic and environmental factors in childhood allergy. PLoS One. 2013;8(11):e80080. doi:10.1371/journal.pone.0080080.

38. Folorunso S, Adeyemo A. Alleviating classification problem of imbalanced dataset. Afr J Comput ICT. 2013;6:2.

39. Bekkar M, Alitouche TA. Imbalanced data learning approaches review. Int J. 2013;3(4):15-33.

40. Øhrn A, Komorowski J, editors. ROSETTA: a rough set toolkit for analysis of data. Durham: Proc. Third International Joint Conference on Information Sciences, Fifth International Workshop on Rough Sets and Soft Computing (RSSC'97); 1997. March 1-5.

41. Komorowski J. Jan Komorowski's Bioinformatics Lab. 2014. In Repositories$>$ Rosetta at http://bioinficm.uu.se/. Accessed 10 Dec 2014.

42. Komorowski J, Pawlak Z, Polkowski L, Skowron A. Rough sets: a tutorial. Rough fuzzy hybridization: a new trend in decision-making. 1999. p. 3-98.

43. Komorowski J. Learning Rule-Based Models - The Rough Set Approach. In: Anders Brahme, editorin-chief. Comprehensive Biomedical Physics. Vol 6. Amsterdam: Elsevier; 2014. p. 19-39.

44. Price MN, Dehal PS, Arkin AP. FastTree 2-approximately maximumlikelihood trees for large alignments. PLoS One. 2010;5(3):e9490. doi:10. 1371/journal.pone.0009490.

45. Smeenk CA, Wright KE, Burns BF, Thaker AJ, Brown EG. Mutations in the hemagglutinin and matrix genes of a virulent influenza virus variant, A/FM/1/ 47-MA, control different stages in pathogenesis. Virus Res. 1996;44(2):79-95.

46. Liu T, Ye Z. Introduction of a temperature-sensitive phenotype into influenza ANSN/33 virus by altering the basic amino acid domain of influenza virus matrix protein. J Virol. 2004;78(18):9585-91. doi:10.1128/JVI.78. $18.9585-9591.2004$

47. Watanabe K, Handa H, Mizumoto K, Nagata K. Mechanism for inhibition of influenza virus RNA polymerase activity by matrix protein. J Virol. 1996; 70(1):241-7.

48. Akarsu H, Burmeister WP, Petosa C, Petit I, Muller CW, Ruigrok RW, et al. Crystal structure of the M1 protein-binding domain of the influenza A virus nuclear export protein (NEP/NS2). EMBO J. 2003;22(18):4646-55. doi:10.1093/emboj/cdg449.

49. Liu W, Zou P, Ding J, Lu Y, Chen YH. Sequence comparison between the extracellular domain of M2 protein human and avian influenza A virus provides new information for bivalent influenza vaccine design. Microbes Infect/Institut Pasteur. 2005;7(2):171-7. doi:10.1016/j.micinf.2004.10.006.

50. Holsinger $\sqcup$, Lamb RA. Influenza virus M2 integral membrane protein is a homotetramer stabilized by formation of disulfide bonds. Virology. 1991; 183(1):32-43.

51. Jackson D, Hossain MJ, Hickman D, Perez DR, Lamb RA. A new influenza virus virulence determinant: the NS1 protein four C-terminal residues modulate pathogenicity. Proc Natl Acad Sci U S A. 2008;105(11):4381-6. doi:10.1073/pnas.0800482105.

52. Heikkinen LS, Kazlauskas A, Melen K, Wagner R, Ziegler T, Julkunen I, et al. Avian and 1918 Spanish influenza a virus NS1 proteins bind to Crk/CrkL Src homology 3 domains to activate host cell signaling. J Biol Chem. 2008; 283(9):5719-27. doi:10.1074/jbc.M707195200.

53. Min JY, Li S, Sen GC, Krug RM. A site on the influenza A virus NS1 protein mediates both inhibition of PKR activation and temporal regulation of viral RNA synthesis. Virology. 2007;363(1):236-43. doi:10.1016/j.virol.2007.01.038.

54. Hale BG, Kerry PS, Jackson D, Precious BL, Gray A, Killip MJ, et al. Structural insights into phosphoinositide 3-kinase activation by the influenza A virus NS1 protein. Proc Natl Acad Sci U S A. 2010;107(5):1954-9. doi:10.1073/pnas. 0910715107.

55. Hale BG, Jackson D, Chen YH, Lamb RA, Randall RE. Influenza A virus NS1 protein binds p85beta and activates phosphatidylinositol-3-kinase signaling. Proc Natl Acad Sci U S A. 2006;103(38):14194-9. doi:10.1073/pnas.0606109103.

56. Melen K, Kinnunen L, Fagerlund R, Ikonen N, Twu KY, Krug RM, et al. Nuclear and nucleolar targeting of influenza A virus NS1 protein: striking differences between different virus subtypes. J Virol. 2007:81(11):5995-6006. doi:10.1128/JVI.01714-06.

57. Pan C, Cheung B, Tan S, Li C, Li L, Liu S, et al. Genomic signature and mutation trend analysis of pandemic (H1N1) 2009 influenza A virus. PLoS One. 2010;5(3):e9549. doi:10.1371/journal.pone.0009549.

58. Tamuri AU, Dos Reis M, Hay AJ, Goldstein RA. Identifying changes in selective constraints: host shifts in influenza. PLoS Comput Biol. 2009;5(11): e1000564. doi:10.1371/journal.pcbi.1000564.

59. Lipatov AS, Yen HL, Salomon R, Ozaki H, Hoffmann E, Webster RG. The role of the $\mathrm{N}$-terminal caspase cleavage site in the nucleoprotein of influenza $\mathrm{A}$ virus in vitro and in vivo. Arch Virol. 2008;153(3):427-34. doi:10.1007/s00705007-0003-8.

60. Bussey KA, Desmet EA, Mattiacio JL, Hamilton A, Bradel-Tretheway B, Bussey $\mathrm{HE}$, et al. PA residues in the $2009 \mathrm{H} 1 \mathrm{~N} 1$ pandemic influenza virus enhance avian influenza virus polymerase activity in mammalian cells. J Virol. 2011; 85(14):7020-8. doi:10.1128/JVI.00522-11.

61. Desmet EA, Bussey KA, Stone R, Takimoto T. Identification of the N-terminal domain of the influenza virus PA responsible for the suppression of host protein synthesis. J Virol. 2013;87(6):3108-18. doi:10.1128/JVI.02826-12.

62. Jin H, Lu B, Zhou H, Ma C, Zhao J, Yang CF, et al. Multiple amino acid residues confer temperature sensitivity to human influenza virus vaccine strains (FluMist) derived from cold-adapted A/Ann Arbor/6/60. Virology. 2003;306(1):18-24

63. Xu C, Hu WB, Xu K, He YX, Wang TY, Chen Z, et al. Amino acids $473 \mathrm{~V}$ and $598 \mathrm{P}$ of PB1 from an avian-origin influenza A virus contribute to polymerase activity, especially in mammalian cells. J Gen Virol. 2012;93(Pt 3):531-40. doi: 10.1099/vir.0.036434-0.

64. Mehle A, Doudna JA. Adaptive strategies of the influenza virus polymerase for replication in humans. Proc Natl Acad Sci U S A. 2009;106(50):21312-6. doi:10.1073/pnas.0911915106.

65. Yamada S, Hatta M, Staker BL, Watanabe S, Imai M, Shinya K, et al. Biological and structural characterization of a host-adapting amino acid in influenza virus. PLoS Pathog. 2010;6(8):e1001034. doi:10.1371/journal.ppat.1001034.

66. Bussey KA, Bousse TL, Desmet EA, Kim B, Takimoto T. PB2 residue 271 plays a key role in enhanced polymerase activity of influenza $A$ viruses in mammalian host cells. J Virol. 2010;84(9):4395-406. doi:10.1128/JVI.02642-09.

67. Foeglein A, Loucaides EM, Mura M, Wise HM, Barclay WS, Digard P. Influence of PB2 host-range determinants on the intranuclear mobility of the influenza A virus polymerase. J Gen Virol. 2011:92(Pt 7):1650-61. doi:10.1099/vir.0.031492-0.

68. Conenello GM, Zamarin D, Perrone LA, Tumpey T, Palese P. A single mutation in the PB1-F2 of H5N1 (HK/97) and 1918 influenza A viruses 
contributes to increased virulence. PLoS Pathog. 2007;3(10):1414-21. doi:10.1371/journal.ppat.0030141.

69. Burke DF, Smith DJ. A recommended numbering scheme for influenza A HA subtypes. PLoS One. 2014;9(11):e112302. doi:10.1371/journal.pone. 0112302.

70. Caton AJ, Brownlee GG, Yewdell JW, Gerhard W. The antigenic structure of the influenza virus A/PR/8/34 hemagglutinin (H1 subtype). Cell. 1982;31 (2 Pt 1): 417-27.

71. Brownlee GG, Fodor E. The predicted antigenicity of the haemagglutinin of the 1918 Spanish influenza pandemic suggests an avian origin. Philos Trans R Soc Lond B Biol Sci. 2001;356(1416):1871-6. doi:10.1098/rstb.2001.1001.

Submit your next manuscript to BioMed Central and we will help you at every step:

- We accept pre-submission inquiries

- Our selector tool helps you to find the most relevant journal

- We provide round the clock customer support

- Convenient online submission

- Thorough peer review

- Inclusion in PubMed and all major indexing services

- Maximum visibility for your research

Submit your manuscript at www.biomedcentral.com/submit
Biomed Central 